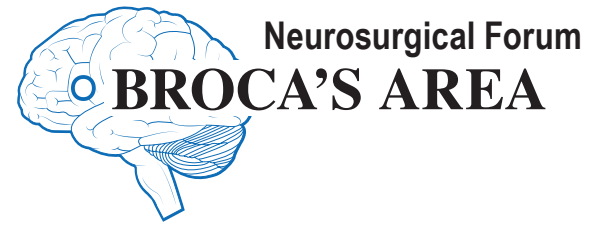

\section{Communication and collaboration in spine neuromonitoring: time to expect more, a lot more, from the neurophysiologists}

\author{
Stan Skinner, MD, ${ }^{1}$ and Francesco Sala, MD² \\ ${ }^{1}$ Abbott Northwestern Hospital, Minneapolis, Minnesota \\ ${ }^{2}$ Institute of Neurosurgery, University Hospital, Verona, Italy
}

$\mathrm{T}$ HIS commentary is addressed to surgeons who order intraoperative neuromonitoring (IONM). In the US, and in some other countries, a certified technologist is commonly supervised by an expert neurophysiologist physician. ${ }^{40}$ US neurophysiologist physicians are often positioned off-site while monitoring several or many cases simultaneously. ${ }^{41}$ As an example of a contrary view that is also held elsewhere in the world, a leading Canadian authority argues, "The vast majority of those involved in IONM in Canada believe, for best practice, the IONM expert should be in the operating room (OR) doing the neurophysiology" (personal communication, David Houlden, University of Ottawa, 2016).

Similarly, the joint guidelines between the Italian Neurosurgical and Clinical Neurophysiology Societies clearly state that the supervising physician should be in the OR or available in-house whenever summoned to the OR. This model is quite popular in other European countries (such as Germany), but other models exist. In Spain, only clinical neurophysiologists are present in the OR and they perform IONM without the support of certified technologists.

Keeping these dissonant approaches in mind, we will: 1) review scholarship that indicates that improved collaboration in the OR is associated with better patient outcomes; 2) demonstrate how IONM injury prevention is predicated on trust-based communication of test results; and 3) propose practical IONM solutions that might better achieve the trusted "expert in the OR" vision.

\section{Communication and the IONM Interventional Cascade}

IONM is a team effort. The technologist and neurophysiologist form the diagnostic testing contingent of the team. The responsible neuromonitorist reports alerts to the surgeon and the anesthesiologist, who decide whether to alter the course of their procedural plan. The intraoperative team as a whole, therefore, participates in a generalized model of test-treatment pathways that affect patient health..$^{11}$ For IONM, this model can be summarized as an interventional cascade: test, interpretation, communication, intervention, and outcome. ${ }^{21}$ With few exceptions, surgeons are imperfectly fluent with the clinical neurophysiology body of knowledge (tests and their interpretation). Neurophysiologists do not independently execute an intraoperative intervention. Therefore, in the event of signal loss, effective neurophysiologist-initiated communication is pivotal to the surgeon's (or anesthesiologist's) decision to act. Favorable patient outcomes must depend on effective communications among co-practicing specialists (surgeon, anesthesiologist, and neurophysiologist). Implicit in this schema is scholarship showing that diagnostic test accuracy per se (within the neurophysiologist's control) is an insufficient basis for clinical effectiveness. ${ }^{11,50}$

Barriers to good decisions and effective patient outcomes exist on either side of the IONM interventional cascade. Surgeons are understandably burdened by a cognitive bias to see their carefully conceived surgical plan through to the end. During IONM reporting, this bias may manifest as a tendency both to construe what is heard in a way that affirms preconceived notions and/or downgrade information that conflicts with "normal" expectations. One of us has summarized this confirmation/expectation bias: 45 "Neurophysiological feedback was generally welcome as long as it reassured the neurosurgeon that everything was going well ('Am I doing OK? Are your evoked potentials stable?'), but was not so well-received whenever there was a change in the evoked potentials that would imply the need to halt or even abandon surgery." And, "The idea of a different professional figure (namely 
a neurologist or clinical neurophysiologist) working hand in hand with the neurosurgeon in the operating room and advising him whether or not his surgical strategy was impairing the well-being of the nervous system was (and still is) something not readily acceptable to neurosurgeons." How much more difficult is the communication task if the neurophysiologist is an unseen, unknown remotely sited "colleague"?

Unfortunately, some supervising neurophysiologists hold themselves above the exigencies of meaningful relationships with their in-room surgeon and anesthesiologist colleagues. They persist in the insular position that IONM amounts to no more than a strictly dispassionate interpretation of waveforms, not unlike a pathologist or radiologist reading a static image. ${ }^{53}$ This is an unfortunate posture by some neurophysiologist physicians. IONM is not the static reading of a test at one point in time, rather it is the ongoing analysis of a data stream over time within a constantly changing surgical context. Furthermore, to presume that an absentee/remote neurologist's opinion may easily sidetrack the surgical plan is naïve and, potentially, dangerously misguided.

The scholarship on communication during high-risk events (such as an IONM alert) indicates: ${ }^{22}$ "The need to establish trust ... is fundamental to the effectiveness of risk communication messages and strategies... Trust, therefore, must be established well in advance of an actual crisis event [italics added]." Vincent Covello, a leading scholar in the risk communication literature, has captured this idea with the apothegm: "They want to know that you care before they care what you know." 5

\section{Spine IONM Evidence: Moving From Prediction to Prevention}

Although the evidence supporting IONM to prevent spinal cord and/or nerve root injury remains mixed, we believe the preponderance of the evidence justifies IONM in many of the varied settings of spine surgery. Several evidential limitations persist, among them: few controlled trials, paradox or bias in the categorization of recovered signal loss in observational studies, unsettled motor evoked potential alert criteria, and the unknowable confounders within and disagreement among recent supersized retrospective data sets. ${ }^{4,32,38,46,54}$ Notwithstanding these limitations, the reliability of any future IONM outcome trials is threatened when the neurophysiologist's role is diminished or neglected. As already described within the interventional cascade, the surgeon's decision to change the course of surgery after an alert crucially depends on the credibility of the interpreter/communicator. In fact, trusted communication determines the efficiency of the link between IONM test accuracy (prediction) and reduced intraoperative injuries (prevention).

A major systematic review has established that IONM can predict neurological injuries. ${ }^{42}$ The combined positive predictive value (PPV) for serious neurological injury was 0.30 . This PPV may seem low until one realizes that in a low prevalence context (risk $\leq 1 \%$ in extradural spine surgery, for example) the PPV will look low even when the test is very accurate. ${ }^{20,54}$ Among the reviewed Class I studies, no false-negative reports were made. However, a prediction analysis that looks at differences in outcomes (no injuries with negative testing, 30\% risk of serious injury with positive testing) incompletely informs us about the role IONM might play in injury prevention. We are left to hope that surgeons-understanding these data when acknowledging an IONM alert-will intervene appropriately. But there is no guarantee of that.

Surgeons' responses to IONM alerts have been studied. ${ }^{62}$ Decisions to respond (or not) to an alert were usually based on context: a recent surgical manipulation, for example, or coincident report from the anesthesiologist (low blood pressure, for example). Only about $50 \%$ of alerts were followed by an intervention. Within the category "true-positive findings without intervention... The surgeon did not react to the information obtained from the IOM, and the patient suffered from a new neurological deficit corresponding to the target of monitoring," listed reasons for inaction included anticipated injury with operative step, manipulation not reversible, and no identifiable reason for IONM change. ${ }^{62}$

A likely factor woven into the surgeons' inability or hesitance to intervene is cognitive bias, predispositions that restrain the surgeon from changing the course of surgery. We have already discussed one of these, i.e., confirmation/ expectation bias. In the event of an alert, deeply instilled beliefs, bias, and previously learned rules of thumb (heuristics) come to mind first. ${ }^{12,25}$ When well-trained professionals (airline pilots and surgeons, for example) are confronted by routine challenges, heuristics permit quick and usually correct decisions. Overreliance on heuristics may limit options during the stress of crisis decision-making. ${ }^{23,49,58}$ At the point of an IONM alarm, the surgeon/decision-maker may also be subject to cognitive errors ${ }^{3,6}$ Checklists may close this decision-making gap during crisis management. Two compelling before/after surgical safety checklist trials convinced both neurosurgical and orthopedic spine groups to generate checklists for IONM alarms., 77,59,64 The orthopedic spine surgery checklist includes a call to "summon" the "senior neurologist or neurophysiologist" after an IONM alarm. ${ }^{59}$ Within many of the surgeon authors' institutions, a trusted in-house neurologist/neurophysiologist is available to be summoned. But an at-hand IONM colleague is not the general rule in the US.

The Scoliosis Research Society has reviewed the use of IONM in 108,419 surgeon-reported cases. The sensitivity for postoperative spinal cord deficit when both somatosensory and motor evoked potentials were recorded was 0.43 . The authors' explanations for poorer than expected IONM sensitivity included variations in "... how [signal changes] are reported to the operating surgeon...."14

This particular explanation by Hamilton (“... how [signal changes] are reported....") gets to the crux of the need to urgently consider and improve the culture of communication between the neurophysiologist and surgeon. In the midst of an IONM alert, it takes a trusted neurophysiologist colleague to resist the surgeon's understandable cognitive bias to complete the surgery as planned. Breaking through this bias demands that the neurophysiologist introduce both crucial neuropathophysiological concepts as well as a coherent probability analysis of context-wedded IONM results. In the absence of trusted communication 
among familiar colleagues, it is very hard to get from IONM diagnostic test accuracy (prediction) to improved outcomes based on injury reduction (prevention).

\section{Medical Error Avoidance in the OR: The Communication Mandate}

The accumulation of man-made disasters in the commercial airline and nuclear industries more than 30 years ago led to the discovery that as many two-thirds of these events were directly related to failed communications among responsible crew members. ${ }^{19}$ More than 20 years after the introduction of Crew Resource Management (CRM) training in commercial aviation, the Institute of Medicine published its findings: tens of thousands of deaths annually were occurring due to potentially preventable medical errors. ${ }^{26}$ Other reports suggested that treatment team miscommunication accounted for twice as many errors as incompetent care..$^{57,63}$ The concern for medical error avoidance has triggered an expansive scholarship whose themes all specify the centrality of robust communication and collaboration among physicians (and nonphysicians as well). ${ }^{2,9,13,16,18,27,30,31,43,48,49,61}$

In ORs, such "non-technical" errors may be initially addressed by the institution of already-cited checklists. . $^{59,64}$ Beyond checklists, intercollegial communication, situational awareness, and means to secure acceptance of an intraoperative cultural change have been suggested by leading authorities..$^{9,27,30}$ Specific training regimens have been created. These include CRM in a medical setting, Systems Engineering Initiative to Patient Safety, and others. ${ }^{9}$ So far, the published trials comparing culturally trained versus untrained surgical "crews" indicates that better OR collaboration can be instilled and postsurgical outcomes can improve after training. ${ }^{10,33,37,44}$ Comparison of OR team training in the culture of communication and collaboration to an untrained propensity-matched cohort demonstrated a $50 \%$ reduced mortality rate in the trained group $(\mathrm{p}=0.01)$. Furthermore, a reduction of 0.5 deaths per 1000 procedures was observed for each additional training quarter, a dose-response correlation $(\mathrm{p}=0.001) .{ }^{37}$

\section{Challenges to Effective IONM Collaboration}

The work of Vitale and colleagues ${ }^{59}$ goes beyond an IONM checklist. By presuming that the expert neurophysiologist can be "summoned" to the OR, the checklist actually establishes the need for collaboration among expert peers: a surgeon/anesthesiologist/neurophysiologist copractice. Once the advantages of a fully collaborating intraoperative team are recognized, the troubling variations in models of IONM care delivery must be confronted.

When IONM staffing patterns were reviewed in 1997, 1-to-1 IONM coverage by credentialed neurophysiologists (versus multiple case management by 1 neurophysiologist) was still the subject of "disagreement and debate." ${ }^{39}$ In view of the exponentially increasing demand for IONM over the last 15 or more years, that debate (in the US) has been settled: 50,000 in 2004, 150,000 in 2010, and current estimates of more than 500,000 cases per year. US neurophysiologist physicians routinely monitor the work of multiple in-room technologists via either an in-house in- tranet or web-based connections from a remote site. ${ }^{41}$ The pressures to accelerate the acceptance of remote IONM are multiple, but 3 are primary: 1) the ease of connections to transmit waveform and basic e-chat data (an informal online communication between the IONM technologist and supervising neurophysiologist); 2) insufficient number of expert neurophysiologists (2000 experts in 1997, 1300 experts in 2009);41 and 3) the obvious financial incentive to cluster as many cases as possible per time epoch. The 2012 decision by the US Center for Medicaid and Medicare Services to eliminate multiple-case Medicare payments has ironically incentivized case clustering to maintain physician income in a reduced reimbursement environment. The incentives to maximize the number of cases per epoch considerably worsen the limits of current remote technology (waveform telemetry and phone/e-chat connections only).

Remote IONM has not been uniformly welcomed:8 "How can an individual outside the OR interpret fluctuating neuromonitoring data and develop explanations of cause? How it is possible to verify an appropriate level of vigilance on the part of the remotely connected professional, particularly if multiple cases are being monitored simultaneously?" Such skepticism may be further fueled by the expanding scholarship on communication, trust, and collaboration in high-risk areas of the hospital. This evidence is recognized within an increasingly robust health safety culture. An extraordinarily heavy burden of proof will be borne by remote professionals who still wish to remain unknown to their patients and unfamiliar to their in-room colleagues. ${ }^{24,48,52,53}$

\section{What Surgeons Should Require of Attending Neurophysiologists}

In an optimal IONM setting, a trusted "senior neurologist or neurophysiologist" can, indeed, be personally "summon[ed]" to the OR in the event of an IONM alarm.59 This fits Nuwer's "nearby and available" model, i.e., the surgeon and in-room technologist enjoy immediate personal availability of an expert neurophysiologist. ${ }^{40}$ That expert is otherwise intranet-connected to a limited number of ORs (usually $\leq 3$ ). In some cases, good decision-making may depend on the expert neurophysiologist's unsolicited physical presence. Mapping of eloquent cortex, plexus, conus-cauda equina, and floor of the fourth ventricle come to mind as possible contexts. When the responsible expert neurophysiologist shows up to sort out difficult troubleshooting problems or offers meaningful advice on pathophysiology, a trusting relationship among peer colleagues is better secured before the next serious IONM event. As a by-product, reports that "all is well" during difficult maneuvers also become more credible.

In the face of mounting evidence that active intercollegial collaboration improves intraoperative safety, surgeons' grudging acceptance of an unseen, unknown expert neurophysiologist warrants critical review. ${ }^{51}$ When a hospital-based "senior neurologist or neurophysiologist" is unavailable, a few less-than-optimal (but potentially acceptable) choices come to mind for surgeons who demand better credibility of their IONM service. These choices begin to approach "expert in the OR" and may ultimately 
prove more doable and attractive under the current economically stressed reimbursement arrangements.

\section{Tele-IONM Option}

One option is to fully integrate the "remote" supervising professional through full spectrum telemedicine similar to tele-stroke. "Face-to-face" audiovisual tele-connections with patients and colleagues in stroke evaluation and treatment, for example, have proven as effective as on-site evaluation. ${ }^{34,47,60}$ Moreover, tele-stroke connectivity is significantly more effective than telephone contact in randomized trials. ${ }^{15,35}$ Similar face-to-face connections are possible in IONM. A recent IONM supervision guideline ${ }^{52}$ does refer to "...fully realized telemedicine....as needed or appropriate;" however, the evolving medical error avoidance literature suggests any future revision should include stronger guidance on the importance of improved intraoperative peer communication and collaboration. ${ }^{52}$

Nevertheless, it is unclear just how closely matched tele-stroke is to an anticipated "tele-IONM" paradigm. For example, in tele-stroke there is one point of decision to employ thrombolytics or not, whereas in tele-IONM, a continuous stream of neurophysiological data are integrated with a constantly changing surgical context over time. Therefore, positive evidence proving tele-IONM equivalence to in-hospital IONM may be required. In sum, Dormans' questions would still need to be answered. ${ }^{8}$

\section{Other Options}

Another option would be to substantially upgrade certification and credentialing of the technologist already in the room. Current US certification in neurophysiological intraoperative monitoring (CNIM) specifically disallows diagnostic test interpretation. Any proposed advanced training and certification must include crucial nontechnical standards. The newly defined "technologist/neurophysiologist" must bear sufficient and verifiable credentials (command of the IONM body of knowledge) to assist the surgeon with in-room interpretation and differential causation in the event of an alert. Just as importantly, the technologist/neurophysiologist must be capable of candid and authoritative communication of IONM findings. This approach questions the fundamental argument that IONM represents the practice of medicine. It has been argued that a physician, not a technologist nor any other nonmedical figure, should be responsible for IONM. ${ }^{1}$ On the other hand, it is recognized that in some countries (Canada and the United Kingdom, for example) professionals other than MDs are responsible for IONM. The Canadian Association of Neurophysiological Monitoring has embarked on a formal 2-year program to achieve both technical and nontechnical (interpretive/collaborative) competence. These arrangements will evolve into a Master's program (personal communication, David Houlden, University of Ottawa, 2016). A similar strategy of upgrading the competence of IONM technologists to interpretative skills, through a Master's program, has been, and still is, a matter of debate in Italy as well. This process will require a rigorous approach and will need a careful evaluation as it questions the definition of IONM as the practice of medicine.

Thus far, there are no data proving that one approach results in a better patient outcome than the other. The challenges associated with advanced credentialing/certification of CNIM technologists in the US are formidable, but the status-quo alternative is unacceptable.

As a variant, the surgeon could be responsible for IONM, if he or she can rely on a properly trained technologist/neurophysiologist who bears proper IONM signal acquisition and interpretation skills. Surgeon-supervised IONM has been sanctioned in the US. ${ }^{1}$ However, there is an advantage in the strict separation of the expert bodies of knowledge among peers (surgeon, anesthesiologist, and neurophysiologist). The cognitive biases particular to each discipline can be negotiated and acted upon more thoughtfully at the moment of critical decision-making, especially in truly collaborative settings. ${ }^{9}$

Semi-automated IONM devices have been widely marketed. The obvious predicate devices are instruments used by otolaryngologists for limited mapping of the facial or recurrent laryngeal nerves during skull base surgery and thyroidectomy, respectively. Spine surgeons are already independently making real-time decisions based on Carm, ultrasonography, intraoperative CT, and stealth navigation technologies. The desire by some surgeons to extend their diagnostic purview to the limited task of pedicle screw threshold testing, for example, is understandable. However, devices do not replace thinking about what you are doing. Therefore, "The attempt to radically reduce the [pedicle screw] data stream to the perfunctory display of an all-encompassing 'number' or 'stoplight' is unlikely to be helpful." 55 Inexpert use of semi-automated or, for that matter, any device during the very complex task of multimodality IONM invites disaster. ${ }^{28,29,36,56}$ In general, monitoring techniques require a higher degree of expertise than mapping techniques. Intraoperative neurophysiology of the spinal cord is essentially based on monitoring techniques. Therefore, to foster the idea that IONM expertise is irrelevant represents a threat to patients and, ultimately, to surgeons as well.

The answer to good IONM in the absence of an in-house expert remains uncertain. Solutions to proper IONM credentialing and models of care will be differentially determined by country, i.e., local IONM societies, statutes, and standards of practice. Full-spectrum tele-IONM may be readily achieved in the US, where robust web-based connections already exist and the predicate tele-stroke model has proven itself. Tele-IONM, despite some evidencebased reservations, promises a level of patient-centered care and professional collaboration that surgeons ordering IONM should routinely expect. Regardless of the approach chosen to address the demand for IONM coverage, the issue of high-quality IONM cannot be negotiable.

\section{Conclusions}

Given the scholarship on the importance of trust and communication and collaboration within ORs, surgeons need not (and probably should not) continue to accept "remote" IONM as generally practiced in the US. The evidence in support of IONM may remain confounded until effective IONM communication and collaboration standards are broadly respected and customarily instituted. The inertia associated with US IONM societies suggests 
that surgeons and their societies-especially in the USmay need to demand creation and implementation of higher standards of communication, situational awareness, and collaboration. It is certainly time to ask much more of the neurophysiologists.

\section{References}

1. American Medical Association: Resolution 201, A-08: Intraoperative Neurophysiologic Monitoring H-410.957. (https://searchpf.ama-assn.org/SearchML/searchDetails. action?uri=\%2FAMADoc\%2FHOD.xml-0-3640.xml) [Accessed January 23, 2017]

2. Awad SS, Fagan SP, Bellows C, Albo D, Green-Rashad B, De la Garza M, et al: Bridging the communication gap in the operating room with medical team training. Am J Surg 190:770-774, 2005

3. Berner ES, Graber ML: Overconfidence as a cause of diagnostic error in medicine. Am J Med 121 (5 Suppl):S2S23, 2008

4. Cole T, Veeravagu A, Zhang M, Li A, Ratliff JK: Intraoperative neuromonitoring in single-level spinal procedures: a retrospective propensity score-matched analysis in a national longitudinal database. Spine (Phila Pa 1976) 39:1950-1959, 2014

5. Covello VT: Risk communication, radiation, and radiological emergencies: strategies, tools, and techniques. Health Phys 101:511-530, 2011

6. Croskerry P: From mindless to mindful practice-cognitive bias and clinical decision making. N Engl J Med 368:24452448, 2013

7. de Vries EN, Prins HA, Crolla RM, den Outer AJ, van Andel G, van Helden SH, et al: Effect of a comprehensive surgical safety system on patient outcomes. N Engl J Med 363:1928 1937,2010

8. Dormans JP: Establishing a standard of care for neuromonitoring during spinal deformity surgery. Spine (Phila Pa 1976) 35:2180-2185, 2010

9. ElBardissi AW, Sundt TM: Human factors and operating room safety. Surg Clin North Am 92:21-35, 2012

10. ElBardissi AW, Wiegmann DA, Henrickson S, Wadhera R, Sundt TM III: Identifying methods to improve heart surgery: an operative approach and strategy for implementation on an organizational level. Eur J Cardiothorac Surg 34:10271033,2008

11. Ferrante di Ruffano L, Hyde CJ, McCaffery KJ, Bossuyt PM, Deeks JJ: Assessing the value of diagnostic tests: a framework for designing and evaluating trials. BMJ 344:e686, 2012

12. Gigerenzer G, Gaissmaier W: Heuristic decision making. Annu Rev Psychol 62:451-482, 2011

13. Haerkens MH, Jenkins DH, van der Hoeven JG: Crew resource management in the ICU: the need for culture change. Ann Intensive Care 2:39, 2012

14. Hamilton DK, Smith JS, Sansur CA, Glassman SD, Ames CP, Berven SH, et al: Rates of new neurological deficit associated with spine surgery based on 108,419 procedures: a report of the scoliosis research society morbidity and mortality committee. Spine (Phila Pa 1976) 36:1218-1228, 2011

15. Handschu R, Littmann R, Reulbach U, Gaul C, Heckmann $\mathrm{JG}$, Neundörfer B, et al: Telemedicine in emergency evaluation of acute stroke: interrater agreement in remote video examination with a novel multimedia system. Stroke 34:2842-2846, 2003

16. Haugen AS, Søfteland E, Eide GE, Sevdalis N, Vincent CA, Nortvedt MW, et al: Impact of the World Health Organization's Surgical Safety Checklist on safety culture in the operating theatre: a controlled intervention study. Br J Anaesth 110:807-815, 2013
17. Haynes AB, Weiser TG, Berry WR, Lipsitz SR, Breizat AH, Dellinger EP, et al: A surgical safety checklist to reduce morbidity and mortality in a global population. N Engl J Med 360:491-499, 2009

18. Helmreich RL: On error management: lessons from aviation. BMJ 320:781-785, 2000

19. Helmreich RL, Merritt AC, Wilhelm JA: The evolution of Crew Resource Management training in commercial aviation. Int J Aviat Psychol 9:19-32, 1999

20. Henderson AR: Assessing test accuracy and its clinical consequences: a primer for receiver operating characteristic curve analysis. Ann Clin Biochem 30:521-539, 1993

21. Howick J, Cohen BA, McCulloch P, Thompson M, Skinner SA: Foundations for evidence-based intraoperative neurophysiological monitoring. Clin Neurophysiol 127:8190, 2016

22. Infanti J, Sixsmith J, Barry MM, Núñez-Córdoba J, Oroviogoicoechea-Ortega C, Guillén-Grima F: A Literature Review on Effective Risk Communication for the Prevention and Control of Communicable Diseases in Europe. Stockholm: European Centre for Disease Prevention and Control, 2013 (http://ecdc.europa.eu/en/publications/ publications/risk-communication-literary-review-jan-2013. pdf) [Accessed January 23, 2017]

23. Janis IL, Mann L: Decision Making: A Psychological Analysis of Conflict, Choice, and Commitment. New York: Free Press, 1977

24. Joint Commission on Accreditation of Healthcare Organizations: Patient safety systems. CAMH Update 2:PS1-PS-54, 2016 (https://www.jointcommission.org/assets/1/18/ PSC_for_Web.pdf) [Accessed January 23, 2017]

25. Kahneman D: Thinking, Fast and Slow. New York: Farrar, Straus and Giroux, 2011

26. Kohn LT, Corrigan JM, Donaldson MS (eds): To Err Is Human: Building a Safer Health System. Washington, DC: National Academy Press, 2000

27. Leonard M, Graham S, Bonacum D: The human factor: the critical importance of effective teamwork and communication in providing safe care. Qual Saf Health Care 13 (Suppl 1):i85-i90, 2004

28. Lieberman JA, Berven S, Gardi J, Hu S, Lyon R, MacDonald $\mathrm{DB}$, et al: To the editor. False-negative transcranial motor evoked potentials during scoliosis surgery causing paralysis. Spine (Phila Pa 1976) 35:717-718, 2010 (Letter)

29. Lieberman JA, Berven S, Gardi J, Hu S, Lyon R, MacDonald DB, et al: Rebuttal. False-negative transcranial motor evoked potentials during scoliosis surgery causing paralysis. Spine (Phila Pa 1976) 35:718-720, 2010 (Letter)

30. Lingard L, Espin S, Whyte S, Regehr G, Baker GR, Reznick $\mathrm{R}$, et al: Communication failures in the operating room: an observational classification of recurrent types and effects. Qual Saf Health Care 13:330-334, 2004

31. Lingard L, Reznick R, Espin S, Regehr G, DeVito I: Team communications in the operating room: talk patterns, sites of tension, and implications for novices. Acad Med 77:232237,2002

32. Macdonald DB, Skinner S, Shils J, Yingling C: Intraoperative motor evoked potential monitoring - a position statement by the American Society of Neurophysiological Monitoring. Clin Neurophysiol 124:2291-2316, 2013

33. McCulloch P, Mishra A, Handa A, Dale T, Hirst G, Catchpole K: The effects of aviation-style non-technical skills training on technical performance and outcome in the operating theatre. Qual Saf Health Care 18:109-115, 2009

34. Meyer BC, Lyden PD, Al-Khoury L, Cheng Y, Raman R, Fellman R, et al: Prospective reliability of the STRokE DOC wireless/site independent telemedicine system. Neurology 64:1058-1060, 2005

35. Meyer BC, Raman R, Hemmen T, Obler R, Zivin JA, Rao 
$\mathrm{R}$, et al: Efficacy of site-independent telemedicine in the STRokE DOC trial: a randomised, blinded, prospective study. Lancet Neurol 7:787-795, 2008

36. Modi HN, Suh SW, Yang JH, Yoon JY: False-negative transcranial motor-evoked potentials during scoliosis surgery causing paralysis: a case report with literature review. Spine (Phila Pa 1976) 34:E896-E900, 2009

37. Neily J, Mills PD, Young-Xu Y, Carney BT, West P, Berger $\mathrm{DH}$, et al: Association between implementation of a medical team training program and surgical mortality. JAMA 304:1693-1700, 2010

38. Ney JP, van der Goes DN, Nuwer MR: Does intraoperative neurophysiologic monitoring matter in noncomplex spine surgeries? Neurology 85:2151-2158, 2015

39. Nuwer JM, Nuwer MR: Neurophysiologic surgical monitoring staffing patterns in the USA. Electroencephalogr Clin Neurophysiol 103:616-620, 1997

40. Nuwer MR: Overview and history, in Daube JR, Mauguière F, Nuwer MR (eds): Handbook of Clinical Neurophysiology, Intraoperative Monitoring of Neural Function. Vol. 8. Amsterdam: Elsevier, 2008, pp 2-6

41. Nuwer MR, Cohen BH, Shepard KM: Practice patterns for intraoperative neurophysiologic monitoring. Neurology 80:1156-1160, 2013

42. Nuwer MR, Emerson RG, Galloway G, Legatt AD, Lopez J, Minahan R, et al: Evidence-based guideline update: intraoperative spinal monitoring with somatosensory and transcranial electrical motor evoked potentials: report of the Therapeutics and Technology Assessment Subcommittee of the American Academy of Neurology and the American Clinical Neurophysiology Society. Neurology 78:585-589, 2012

43. Reeves S, Lewin S, Espin S, Zwarenstein M: Interprofessional Teamwork for Health and Social Care. Chichester, UK: Wiley-Blackwell, 2010

44. Sacks GD, Shannon EM, Dawes AJ, Rollo JC, Nguyen DK, Russell MM, et al: Teamwork, communication and safety climate: a systematic review of interventions to improve surgical culture. BMJ Qual Saf 24:458-467, 2015

45. Sala F: Intraoperative neurophysiology is here to stay. Childs Nerv Syst 26:413-417, 2010

46. Sala F, Palandri G, Basso E, Lanteri P, Deletis V, Faccioli F, et al: Motor evoked potential monitoring improves outcome after surgery for intramedullary spinal cord tumors: a historical control study. Neurosurgery 58:1129-1143, 2006

47. Schwab S, Vatankhah B, Kukla C, Hauchwitz M, Bogdahn U, Fürst A, et al: Long-term outcome after thrombolysis in telemedical stroke care. Neurology 69:898-903, 2007

48. Sevdalis N, Hull L, Birnbach DJ: Improving patient safety in the operating theatre and perioperative care: obstacles, interventions, and priorities for accelerating progress. Br J Anaesth 109 (Suppl 1):i3-i16, 2012

49. Sexton JB, Thomas EJ, Helmreich RL: Error, stress, and teamwork in medicine and aviation: cross sectional surveys. BMJ 320:745-749, 2000

50. Siontis KC, Siontis GCM, Contopoulos-Ioannidis DG,
Ioannidis JP: Diagnostic tests often fail to lead to changes in patient outcomes. J Clin Epidemiol 67:612-621, 2014

51. Skinner S: Patient-centered care model in IONM: a review and commentary. J Clin Neurophysiol 30:204-209, 2013

52. Skinner SA, Cohen BA, Morledge DE, McAuliffe JJ, Hastings JD, Yingling CD, et al: Practice guidelines for the supervising professional: intraoperative neurophysiological monitoring. J Clin Monit Comput 28:103-111, 2014

53. Skinner SA, Cohen BA, Morledge DE, McAuliffe JJ, Hastings JD, Yingling CD, et al: To the editor. J Clin Monit Comput 29:317-318, 2015 (Letter)

54. Skinner SA, Holdefer RN: Intraoperative neuromonitoring alerts that reverse with intervention: treatment paradox and what to do about it. J Clin Neurophysiol 31:118-126, 2014

55. Skinner SA, Rippe DM: Threshold testing of lumbosacral pedicle screws: a reappraisal. J Clin Neurophysiol 29:493501,2012

56. Skinner SA, Rippe DM: Re: False-negative transcranial motor-evoked potentials during scoliosis surgery causing paralysis. Spine (Phila Pa 1976) 35:721-722, 2010 (Letter)

57. Solet DJ, Norvell JM, Rutan GH, Frankel RM: Lost in translation: challenges and opportunities in physician-tophysician communication during patient handoffs. Acad Med 80:1094-1099, 2005

58. Starcke K, Brand M: Decision making under stress: a selective review. Neurosci Biobehav Rev 36:1228-1248, 2012

59. Vitale MG, Skaggs DL, Pace GI, Wright ML, Matsumoto $\mathrm{H}$, Anderson RC, et al: Best practices in intraoperative neuromonitoring in spine deformity surgery: development of an intraoperative checklist to optimize response. Spine Deform 2:333-339, 2014

60. Wang S, Lee SB, Pardue C, Ramsingh D, Waller J, Gross H, et al: Remote evaluation of acute ischemic stroke: reliability of National Institutes of Health Stroke Scale via telestroke. Stroke 34:e188-e191, 2003

61. Wheelan SA, Burchill CN, Tilin F: The link between teamwork and patients' outcomes in intensive care units. Am J Crit Care 12:527-534, 2003

62. Wiedemayer H, Fauser B, Sandalcioglu IE, Schäfer H, Stolke D: The impact of neurophysiological intraoperative monitoring on surgical decisions: a critical analysis of 423 cases. J Neurosurg 96:255-262, 2002

63. Wilson RM, Runciman WB, Gibberd RW, Harrison BT, Newby L, Hamilton JD: The quality in Australian health care study. Med J Aust 163:458-471, 1995

64. Ziewacz JE, Arriaga AF, Bader AM, Berry WR, Edmondson L, Wong JM, et al: Crisis checklists for the operating room: development and pilot testing. J Am Coll Surg 213:212-217, 217.e1-217.e10, 2011 (Erratum in J Am Coll Surg 215:310, 2012)

\section{Disclosures}

Dr. Skinner has a royalty-bearing relationship with Medtronic, Inc. 